\title{
A Study on the Education of Data Analysing Technology for the Urban Planning in the New Data Environment
}

\author{
Yan Xiao, Hui Sun, and Bin Luan
}

\begin{abstract}
The evolution of the internet and data technologies has formed the new impetus for rapid development of the architecture and urban planning, more broadly, for relative industries and educations. New paradigms and research methodologies are established for the urban planning and designing in this new environment and spatial quantitative analysis has become an indispensable part of the designing process. Using internet thinking to explore possible innovations in teaching methods has become a hotspot of the educators in Urban Planning. This paper has attempted to propose new teaching methods, which is essential in the new environment for the education of Architecture and Urban Planning, from the perspectives of quantitative cognitions on the architectures and city layouts. The outcomes of this research could be considered as a reference for the featured professional education in Architecture.
\end{abstract}

Index Terms-Big data, quantitative analysis, space syntax, digital design.

\section{INTRODUCTION THE IMPACT OF NDE ON URBAN PLANNING}

Currently, with the innovative leapfrogging development of the internet and information industry, the concept of "Internet plus initiative" has been extensively accepted and applied in various industries and initiates the era of big data [1]. Being parallel with this, there can be seen significant revolutions in the paradigms and methodologies for Architecture and Urban Designing, which has become one hotspot for the academia of Architecture. Under the new data environment (NDE), the interdisciplinary utilisation of state-of-the-art data analysing technologies into education of Urban Planning will become one of the significant contents that educators become interested in.

\section{THE IMPACT OF NDE ON URBAN PLANNING}

\section{A. Concept and Knowledge Renewal}

The explosive increment of information has been constantly influencing the production, operation and life in one city and thus generates many related concepts about cities. It can be confirmed that the era of bit data has come, from the Information City in the 1980s, the Intelligent City

Manuscript received May 30, 2018; revised August 15, 2018. Supported by "China Postdoctoral Science Foundation" (Grant No.: 2016M600203); supported by "the Fundamental Research Funds for the Central Universities".

The authors are with the School of Architecture and Fine Art, Dalian University of Technology, China (e-mail: xiaoyan@dlut.edu.cn, sunliang@dlut.edu.cn). and Digital City in the 1990s, to the Smart City in the current days.

The smart city reflects the profound impacts on the city life from the big data. It detects analyses and integrates critical information of the running of cities with the help of information and communication technology, so as to provide quick and smart response to the demands of the normal life in one city and guarantee that the cities can be operated in an intelligent way (see Fig. 1). The big-data technology plays a significant role in the planning and construction of one city; for instance, it could provide data supports for traffic planning and comprehensive evaluation, economical information acquisitions, e-government affairs and citizen-beneficial policy making

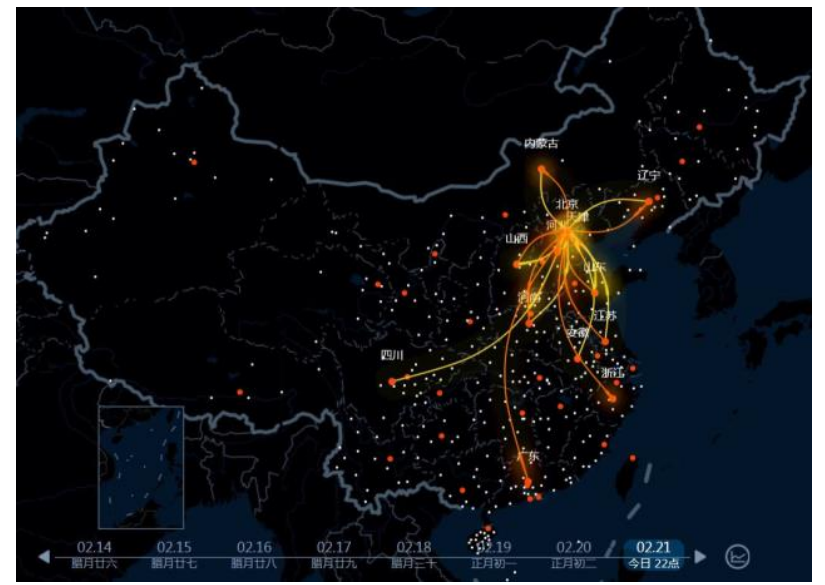

Fig. 1. Visual analysis of urban population migration based on big data in urban planning education

\section{B. Innovations of the Quantitative Study Methodology}

Researchers have started quantitative study on the urban space and architectural environment with the generation of new concepts and changes in research aspects; the innovation in research methodologies emerges as the times require. When being confronted with a large amount of complicated data, the critical prerequisite becomes to categorise and to select the vital and useful information for quantitative analysis. Data-mining, for the present time, is to solve this problem, i.e. extracting potentially valuable information from big data, which is of great significance for the applied technologies and has broad application prospects in scientific domains.

Being enlightened by the big data and data mining, researchers in Urban Planning have proposed specific methodologies for research and design (see Fig. 2). The innovative methodology aims to offer quantitative supports for research and applications of urban planning and to provide scientific supports for the design and planning, so as 
to guarantee the reliability of the design through data validation. The Future Transportation Lab in Shenzhen Urban Transport Planning Centre has carried out experiments and academic studies through data mining, urban simulations and open platforms [2].

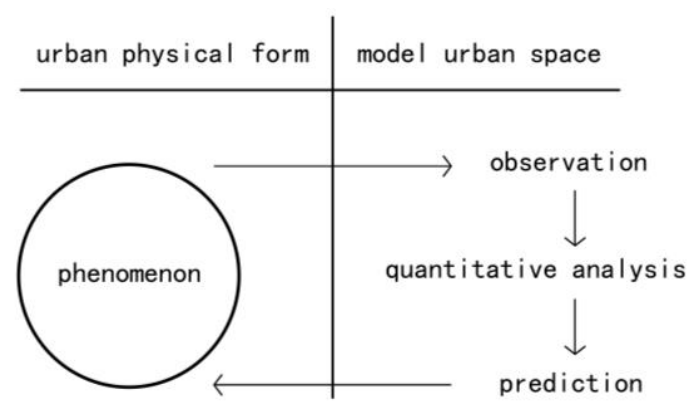

Fig. 2. Quantitative analysis of urban problems.

\section{Applications of Information Technology}

Another revolution in urban planning that comes along with the era of big data is the extensive application of computer science (CS) and information technology (IT). Through the development over the past half century, advanced digital technologies, e.g. CS, IT and the internet techniques, have been extensively utilised in many areas of the urban planning. The GIS (Geographic Information System) is a representative type of urban information technology, which offers to collect, analyse and process the required information for urban planning [3]. These kinds of technology have significantly changed the internal information flows of the Urban Planning and the information feedback mechanisms between Urban Planning organisations and the society. Accordingly, a further and profound impact is generated on the management mode for the planning work. In addition, the big data has also significantly promoted the development of the urban information technology: the EPC (Engineering Procurement Construction) System Network and the utilisation of sensors provide abundant data for the information technology; cloud computing reduces the requirement for data storages and the cost for hardware investment; and the use of big data and relative technology can make the storage of urban data more convenient and efficient

\section{DOMESTIC SituATION OF DOMESTIC EDUCATION}

The current teaching modes of data analysing courses for undergraduate education can be categorised into three types:

\section{A. Independent Software Training Courses}

In China, Computer Aided Design (CAD) curriculums have always been an indispensable part of the undergraduate education for Urban Planning. In the era of big data, however, the shock from complicated data has significantly impacted the architecture and urban planning industry. In this situation, domestic universities have initiated related courses surrounding data analysing software for undergraduate students, among which the ArcGISTM and BIM (Building Information Modelling) are the commonly used. The software training courses are usually tightly arranged in a relatively short period, aiming to train the student to skillfully use the software. However, unlike the designing software such as Sketchup or CAD, the data analysing software requires users' research thinking, which cannot be simply obtained from some training courses [4].

\section{B. Preliminary Applications in Curriculum Design}

On the basis of the software training courses, one significant approach to strengthening students' skills in using software is to integrate software utilisations into specialised training courses. Through these courses, the use of data analysing technologies in the courses can nurture students' ability in quantitative analysis and design and the students would have a deeper understanding of the software and its running mechanism. However, this type of courses necessitates teachers with high proficiency software utilisations, so there is requirement for teachers with the expertise in both design and data analyzing [5].

\section{Skill Strengthening through Design Practice}

Design practice is a vital process for undergraduate students major in urban planning to get acquaintance to the operating mechanism of the design industry. For recent years, students have had more opportunities to get access to practical designing through the practice courses, with the broad applications of data analysing technologies in the design industry. In the environment where the curriculums and practice design are integrated, students can have more precise cognitions about the current status of this area and lay solid foundations for their future careers; however, there have not been as many opportunities in the current situation [6].

\section{Problems in the Current Teaching Mode}

To conform the changes of the data environment, a large number of universities have adjusted their curriculum settings based on their own curriculum system and teaching characteristics. Some universities added courses about quantitative cognitions and data analysing technologies into their original curriculum system, but problems still exist, as depicted below.

\section{A. The Lack of Theoretical Knowledge}

As the internet technology has gradually entered the era of big data, related courses about has been established for the undergraduate education of architecture and urban planning. Currently, the Spatial Syntax [7], ArcGIS and BIM are frequently arranged courses as the computer aided tools for data analyzing (see Fig. 3 and Fig. 4). These courses are usually tightly arranged in a very short period of time, aiming to rapidly increase students' proficiency in using software. However, the contents of scientific methodologies and research thinking behind the software are rarely mentioned in these courses, e.g. the nature of spatial complexities, formation mechanisms of the individual spatial tissue, coupling effects of the spatial elements and their associations with the global distributions.

\section{B. The Ossification of Curriculum Settings}

Most universities, in China, have derived the Urban Planning from the Architecture; In this situation, curriculum 
settings for this major always pay more attention on the professional trainings related to the shapes, global colourings and picture composing for buildings and cities. Therefore, software training courses such as applications of CAD and Sketchup applications are considered as significant contents in the education while the data analysing and related technological trainings are the weak in the curriculum settings. By contrast, situations in western countries are quite different. The applications of data analysing technologies in macro analysis and spatial aided analysis are vital and significant in the curriculum settings. In the meanwhile, there is a lack of flexibility in the curriculum settings for most universities, forming an obliterate teaching environment. The curriculums that are in lack of data analysing courses therefore failed to meet the requirement of development in the new environment.
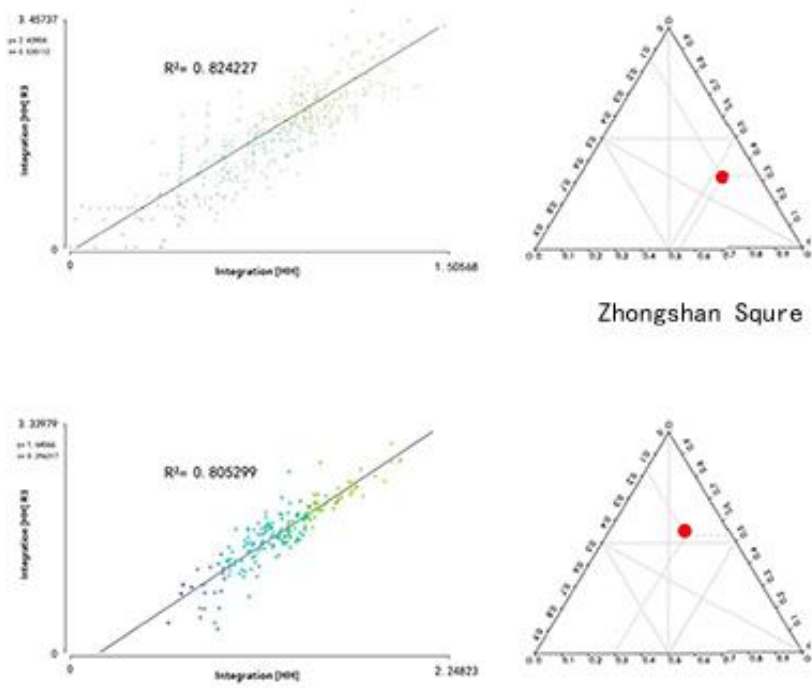

Huanan Squre
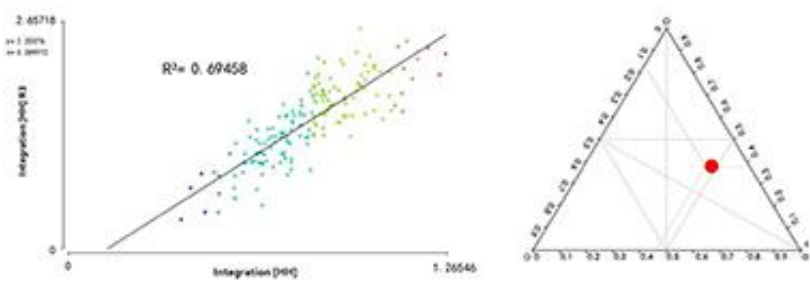

Qixianling

Fig. 3. Student work, quantitative analysis of urban streets based on space syntax.

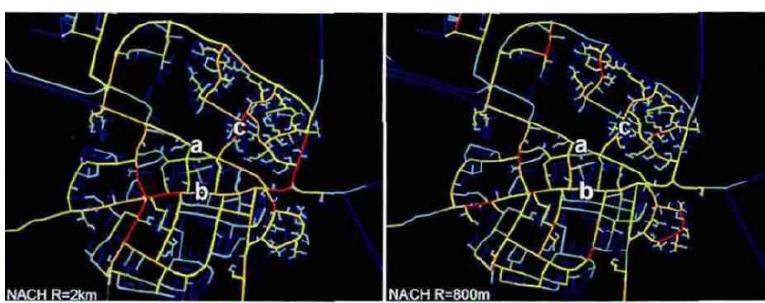

Fig. 4. Quantitative analysis of urban morphology based on space syntax.

\section{Hysteresis in the Education}

At present, the conditions for spatial phenomena have been increasingly complex, along with which are the continuous expansion of space scales and rapid development in digital IT and CS. The research and analysis of the architectures and urban spaces has therefore stepped into a new stage with deeper and more systematic research methodologies. A series of new technologies for related studies has been established, e.g. the data augmented design (DAD) proposed by the Beijing City Lab. Many research organisations working on the quantitative space analysis have also been established, such as the Collaborative Innovation Centre of the Institute of Urban Planning and Design in Nanjing University and Laboratory of Sustainable Smart City in Tongji University. These institutions and organisations focus on not only quantitative descriptions of urban spaces, but also the impact on individual spatial experiences from the space patterns. However, the hysteresis in technological educations has significantly deterred people with conventional expertise in urban planning to adapt to the new demands in the big data era [8].

\section{IMPROVEMENTS TOWARDS DIGITAL DESIGN}

With the initiating of big data era, there has been urgent requirement of people with expertise of data analysis for urban planning and design. The future education in architecture should be able to train students into professionals.

\section{A. Developing Rational Thinking}

Conventional education models for architecture and urban planning are usually bounded by paradigm of aesthetic forms resulting in the problem that logical inductions are always replaced by intuitional judgment. In this situation, the courses tend to be over divergent in thinking modes and also rely too much on the so called inspirations and subjective experiences, which excessively pursue the beauty of patterns and artistry. Therefore, it is an urgent requirement to introduce rational thinking into architecture education. The course setting should be able to guide students in establishing reasonable correlations between spatial phenomena of materials and models, and in increasing their ability for extracting and processing data and corresponding deciphering, predicting and evaluating abilities. As a quantisation approach, the modelling of urban spaces usually requires knowledge of statistics and mathematics to describe, predict and evaluate the material spaces, based on which further curriculum settings can be discussed, validated and optimised.

\section{B. Emphasis of Thematic Studies}

The course of architecture and urban planning has added fundamental contents of data aided design, forming a distinctive topic for teaching. Surrounding the digital design and analysis, topic selections for the course focus on the dynamic balances between research frontiers and practical applications in Urban Planning A series of courses has been set up for spatial cognition and design, covering contents from internal spaces and external fields to the streets and blocks in one city. Therefore, an in-depth and intact research could be attained for the education of Architecture and Urban Planning. At the same time, by combining case studies, the 
basic forms of urban spaces are analysed and evaluated by use of computational approaches. The result validations are carried out using a series of quantitative indices that could also summarise the formative laws, reveal problems and search for solutions to the related problems. Thematic design courses summarise experiences in architecture and urban planning and elucidate the problems of research objects through a large amount of case studies. Based on these case studies, the ultimate goal for this study is to help building up students' abilities in excavating, systematically analysing and solving problems in related areas.

\section{Reinforcement of Technical Supports}

In the NDE, comprehensive and diversified software technologies are the essential elements for quantitative analysis of the architectural and urban spaces. The Space Syntax software should be with features such as simple operations and widely applications, and be able to cover explicit problem guides and to simplify the data collection process. Besides, it is necessary to add contents, which is related to on-line data collection and big data cleaning, into courses as a fundamental practice for students. The data processing and visualisation functions in MATLAB are ideal tools for students getting access to and analysing the data from urban spaces; therefore, it is very suitable to introduce this software and related toolboxes into the course. By use of these tools, the quantitative descriptions of urban spaces could be achieved via the visualising performance of the data.

Besides the comprehensive understanding of the industry, in designing the courses, a significant means is to hire experts from enterprises as supervisors to accelerate the transformation of education in Architecture and Urban Planning. Moreover, in actual projects, supervisors from enterprises with the expertise of data analysing technology could train students with relative skills in a more intact and systematic way. The auxiliary teaching should be introduced into different stages of the undergraduate education, in order to make students keep in pace with the state-of-the-art development of the industry and get them ready for entering the design industry.

\section{Enrichment of Curriculum System}

Facing with the new normal of technology and the NDE, the curriculum setting should focus on the interdisciplinary education of different areas and generate inclusive cognitions and ideologies. Based on the knowledge classification, the big data technology should be categorised into tool knowledge for the education of Architecture and Urban Planning. Along with this is that the analysing technologies for these areas have gradually become the structural knowledge of designers. Related courses have significantly influenced the professional qualities of students and their future careers. Therefore, it is very necessary to add contents of related expertise into the undergraduate curriculums.

The professional knowledge structure is optimised by accomplishing the curriculum system. The cultivation of designers with interdisciplinary expertise could also be achieved through these courses. Therefore, adjustment and changes in the curriculum settings are always essential for curriculums keeping up to date. Moreover, the curriculum setting should also emphasise the fundamental skills of students and encourage the course integrations of quantitative analysis for urban spaces and architecture designing, forming a teaching idea that integrates designing courses with knowledge of digital analysis. Meanwhile, the curriculum settings have also encouraged using interdisciplinary methods which contain theories and research methods in Complexity Science, Urban Planning, Architecture, Urban Designing, Urban Geography, etc.

\section{CONCLUSION}

In the era of "Internet plus initiative", data analysing can be seen as a fundamental stage for general design work. With the help of the courses of quantitative analysis for urban spaces, effective methods can be established for students to overcome conventional thinking modes in the designing process in urban planning, which are in a simple, linear and purely material-focused mode. Instead, professional analysis and planning modes are established for system and quantitative analysis and through the courses, students are expected to carry out scientific thinking and rational analysis from the spatial perspective, use these technologies into aided design. Ultimately, a new education mode can be constructed, which is an ability-oriented diversification and systematisation form.

Educators in urban planning have been exploring new teaching methods and means by adequately integrating the courses with the state-of-the-art research projects. In the NDE, the information technologies are used in teaching practices and scientific research projects. The research about intersecting education is strengthened by integrating new technologies into the teaching practices. Accordingly, it becomes a significant process to establish a novel and reasonable education system and to accumulate teaching experiences in fostering interdisciplinary experts to meet the market requirement. To follow the trend of development in urban planning, as a modern subject, it is necessary to explore novel and reliable education models. This is also a positive attitude towards the current big data environment.

\section{ACKNOWLEDGMENT}

Thanks are due to Professor Hui Sun and Professor Jiang Liang for their instructive advice and useful suggestions on the thesis and to Professor Jun Cai for valuable discussion. In addition, the authors wish to thank Graduate School of Dalian University of Technology for their special fund on graduate student affairs. This work was supported in part by their support.

\section{REFERENCES}

[1] J. Carlson, M. Fosmire, C. C. Miller et al., "Determining data information literacy needs: A study of students and research faculty," Portal Libraries \& the Academy, vol. 2, pp. 629-657, Nov 2011.

[2] J. M. Davidson, "Quantitative reasoning in the planning curriculum," Journal of Planning Education and Research, vol. 6, pp. 30-36, 1986.

[3] C. K. Contant and D. J. Forkenbrock, "Planning methods: An analysis of supply and demand," Journal of Planning Education and Research, vol. 69 , pp. 10-21, 1986 .

[4] A. I. Frank, "Three decades of thought on planning education," Journal of Planning Literature, vol. 21, pp. 15-67, 2006. 
[5] D. J. Forkenbrock, "Planning methods: What can and what should we teach," Journal of Planning Education and Research, vol. 6, pp. 9-10, 1986.

[6] J. Friedmann, "The core curriculum in planning revisited," Journal of Planning Education \& Research, vol. 15, pp. 89-104, 1996.

[7] B. Hillier and J. Hanson, Social Logic of Space, 1st ed. London, U.K.: Cambridge University Press, 1984, ch. 3, pp. 55-59.

[8] Q. Sheng and N. Liu, "Comparing the use of actual space and virtual space: A case study on Beijing's Wangfujing area," The $10^{\text {th }}$ Space Syntax Symposium, pp. 50-52, 2015.

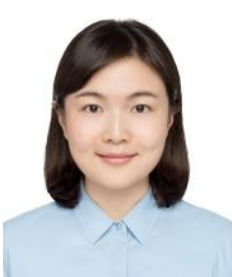

Yan Xiao received her bachelor of urban planning from Dalian University of Technology, China in 2009; she received her MSc degree from University of Technology, China in 2012 and $\mathrm{PhD}$ in architectural design and theory at Dalian University of Technology, China in 2015. She completed two-year postdoc training at Faculty of Infrastructure Engineering Dalian University of Technology in
2017. She is a lecturer at School of Architecture and Fine Art, Dalian University of Technology, Dalian, China.

Hui Sun received his bachelor of architecture from Tsinghua University, China in 1991; he received his MSc degree of urban planning and design from Tsinghua University, China in 1993 and MSc degree of urban planning and design at University of Cincinnati, USA in 1996. He is a professor at School of Architecture and Fine Art, Dalian University of Technology, Dalian, China

Bin Luan received his bachelor of architecture from Dalian University of Technology, China in 2005; he received his MSc degree of urban planning and design from University of Technology, China in 2008. He is a lecturer a School of Architecture and Fine Art, Dalian University of Technology, Dalian, China. 